\title{
Comparative transcriptome analysis uncovers regulatory roles of long non- coding RNAs involved in resistance to powdery mildew in melon
}

\author{
Chao Gao ${ }^{1 * \dagger}$, Jianlei Sun ${ }^{1 \dagger}$, Yumei Dong ${ }^{1}$, Chongqi Wang ${ }^{1}$, Shouhua Xiao ${ }^{1}$, Longfei Mo $^{2}$ and Zigao Jiao ${ }^{{ }^{*}}$
}

\begin{abstract}
Background: Long non-coding RNAs (IncRNAs) are a class of non-coding RNAs with more than 200 nucleotides in length, which play vital roles in a wide range of biological processes. Powdery mildew disease (PM) has become a major threat to the production of melon. To investigate the potential roles of IncRNAs in resisting to PM in melon, it is necessary to identify IncRNAs and uncover their molecular functions. In this study, we compared the IncRNAs between a resistant and a susceptible melon in response to PM infection.
\end{abstract}

Results: It is reported that 11,612 IncRNAs were discovered, which were distributed across all 12 melon chromosomes, and $>85 \%$ were from intergenic regions. The melon IncRNAs have shorter transcript lengths and fewer exon numbers than protein-coding genes. In addition, a total of 407 and 611 IncRNAs were found to be differentially expressed after PM infection in PM-susceptible and PM-resistant melons, respectively. Furthermore, 1232 putative targets of differently expressed IncRNAs (DELs) were discovered and gene ontology enrichment (GO) analysis showed that these target genes were mainly enriched in stress-related terms. Consequently, co-expression patterns between LNC_018800 and CmWRKY21, LNC_018062 and MELO3C015771 (glutathione reductase coding gene), LNC_014937 and CmMLO5 were confirmed by qRT-PCR. Moreover, we also identified 24 IncRNAs that act as microRNA (miRNA) precursors, 43 IncRNAs as potential targets of 22 miRNA families and 13 IncRNAs as endogenous target mimics (eTMs) for 11 miRNAs.

Conclusion: This study shows the first characterization of IncRNAs involved in PM resistance in melon and provides a starting point for further investigation into the functions and regulatory mechanisms of IncRNAs in the resistance to PM.

Keywords: Melon, Comparative transcriptome, Long non-coding RNA, Powdery mildew disease, Expression pattern

\section{Background}

It has been reported that a large portion of the genomic sequences is transcribed [1]. However, only few transcripts encode protein sequences in eukaryotic organisms, suggesting that most transcripts are non-coding

\footnotetext{
*Correspondence: gsuperman114@163.com; zigaojiao5@163.com

${ }^{\dagger}$ Chao Gao and Jianlei Sun contributed equally to this work.

'Shandong Key Laboratory of Greenhouse Vegetable Biology, Shandong Branch of National Improvement Center for Vegetable, Vegetable Science Observation and Experiment Station in Huang huai District of Ministry of Agriculture (Shandong), Institute of Vegetables and Flowers, Shandong Academy of Agricultural Sciences, Jinan 250100, China

Full list of author information is available at the end of the article
}

RNA (ncRNA) [2]. The ncRNA families are composed of small and long non-coding RNA (lncRNAs) based on the length of mature transcripts. Small ncRNAs (approximately 18-30 nucleotides) include microRNAs (miRNAs) and small interfering RNAs (siRNAs), which have been well characterized for their involvement in the regulation of gene expression at transcriptional and post-transcriptional level in almost all eukaryotes [3]. LncRNAs are a class of non-coding RNAs with more than 200 nucleotides in length, which have been demonstrated to participate in the regulation of gene expression during plant growth and development, and various

(c) The Author(s). 2020 Open Access This article is distributed under the terms of the Creative Commons Attribution 4.0 International License (http://creativecommons.org/licenses/by/4.0/), which permits unrestricted use, distribution, and 
stress responses of plants [4-6]. According to their position on the genome, lncRNAs can be classified into long intergenic non-coding RNA (lincRNA), long intronic non-coding RNAs and natural antisense transcripts (lncNATs) [7].

Over the last decades, with the development of highthroughput sequencing, thousands of lncRNAs have been identified in various plant species, such as Arabidopsis, rice, maize, tomato, apple, strawberry and others [8-13]. Many lncRNAs have been functionally characterized in some plants, especially in Arabidopsis and rice, indicating that lncRNAs play critical roles in multiple biological processes including flowering, photomorphogenesis, sex differentiation, and fruit development [14]. In Arabidopsis, 6480 transcripts have been classified as IncRNAs. Among them, one intronic IncRNA transcribed from the first intron of FLOWERING LOCUS C (FLC) and two antisense lncRNAs transcribed from the antisense strand of $F L C$ have been reported to affect the flowing time by negatively regulating FLC expression at epigenetic and posttranscriptional level after cold condition [15]. In rice, it was found that lncRNAs expressed in highly tissuespecific or stage-specific manner, and a set of lncRNAs have been demonstrated to be involved in photoperiodsensitive male sterility and sexual reproduction [16]. In tomato, 490 lncRNAs were significantly up-regulated in ripening mutant fruits rin, and 187 lncRNAs were downregulated, implying that lncRNAs could be involved in the regulation of fruit ripening in tomato [13]. Indeed, silencing of two intergenic lncRNAs in wild-type fruit (lncRNA1459 and lncRNA1840) resulted in an obvious delay of fruit ripening [13].

LncRNAs are also responsive to various biotic and abiotic stresses, and have been confirmed to play significant roles in several biological processes of plant stress responses, such as drought, salt stress and various pathogen stresses $[17,18]$. Drought induced lncRNA (DRIR) in Arabidopsis was expressed at a low level after non-stress conditions but can be significantly activated by drought, salt stress and abscisic acid treatment, which contributes to salt and drought tolerance [19]. In plant-pathogen interactions, lncRNAs also played vital roles in plant's defense system during pathogen infection [20]. In tomato, it was found that slylnc0195 acted as competing endogenous target mimics for miR166 to protect its targets, class III HD-Zip transcription factor genes, and was involved in the resistance against TYLCV infection [18]. Moreover, a set of $F$. oxysporum-induced lncRNAs (15 lncNATs and 20 lincRNAs) were identified in Arabidopsis, and the role of lincRNAs for resistance against $F$. oxysporum was functionally confirmed using T-DNA insertion or RNA-interference knockdown lines [17]. Furthermore, promoter analysis suggested that some of the F. oxysporum-induced lncTARs were direct targets of transcription factors responsive to pathogen attack [17]. Collectively, these studies showed that lncRNAs play important roles during plant growth and development as well as in resisting to various stresses. However, research has not been reported in melon, and little is known about lncRNAs and their potential roles in melon.

Melon (Cucumis melo L.) is an economically important fruit crop that belongs to Cucurbitaceae family, and is susceptible to powdery mildew disease (PM) during the later stage of development [21]. PM is a kind of fungal disease of melon caused by Podosphaera xanthii (Px) or Golovinomyces cichoracearum (Gc), which leads to the decline of melon yield and quality, and PM has severely hindered the development of melon industry [21]. To identify lncRNAs in melon and assess their potential roles in resisting to PM, we used comparative whole transcriptome analysis of PM-resistant and PMsusceptible melon leaves after PM inoculation to identify differentially expressed lncRNAs and investigate lncRNA-mRNA networks. Our results indicated that a large number of lncRNAs were responsive to PM infection, including those that act as endogenous miRNA target or mimics (eTMs), which provided a foundation for further functional analysis of lncRNAs in the resistance to PM.

\section{Results \\ Different phenotype of M1 and B29 after powdery mildew infection}

The occurrence of PM disease was assessed after inoculation with powdery mildew fungus in the greenhouse. As shown in Fig. 1a, no obvious bacterial plaque was observed on M1 leaves at 7 day after powdery mildew infection, while the B29 leaves were wisped with intense mildew (Fig. 1b), indicating the significant difference in resisting to PM between the two genotypes. Previous transcriptome profiling analysis of genes in melon after PM inoculation revealed that the expression of genes involved in the response to biotic stimulus resistance, response to external stimuli, signal transduction, kinase activity, transcription factor activity and plant-pathogen interactions was increased at $24 \mathrm{hpi}$ and high expression levels were maintained to $48 \mathrm{hpi}$, and was subsequently decreased after 48 hpi [22]. Given that the disease resistance response in melon generally occurred before phenotype observed, leaves of both M1 and B29 genotypes were harvested at $24,48 \mathrm{~h}$ post inoculation for further analysis.

\section{Overview of RNA-seq data}

High-throughput sequencing was performed to identify lncRNAs and evaluate their expression in the leaves of PM-resistant lines (M1) and PM-susceptible lines (B29) 


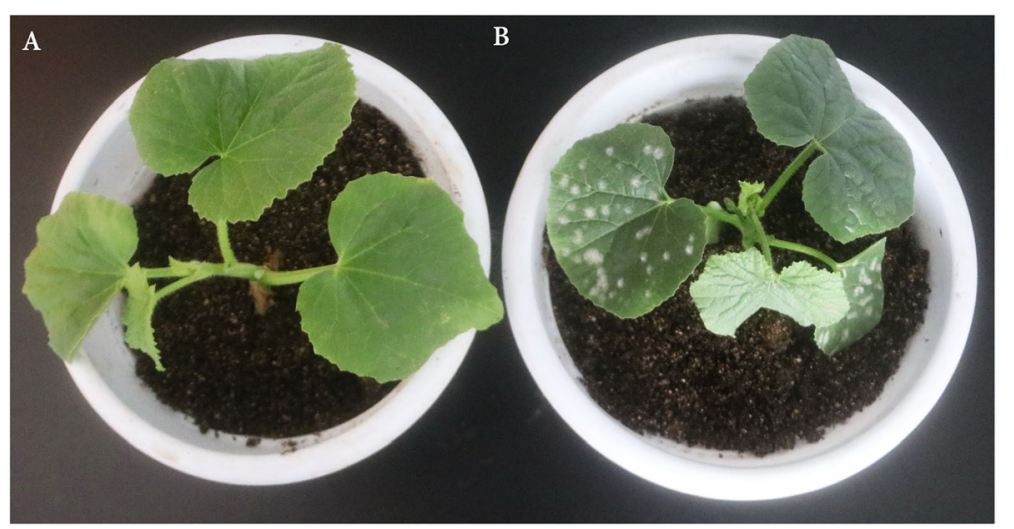

Fig. 1 Different phenotype of two melons observed at 7 day after powdery mildew infection. a: the phenotype of M1; b: the phenotype of B29

infected at 0, 24 and $48 \mathrm{hpi}$. In this study, three biological replicates were used and a total of 18 libraries were sequenced in a $150 \mathrm{bp}$ paired-end module. In all samples, approximately 82.68 to $85.97 \%$ of clean reads were uniquely mapped to the melon reference genome. The rates of genomic match were similar among different samples, suggesting the similar quality of sequence data across the series. Detailed mapping statistics is provided in Additional file 1: Table S1. Based on the expression value of FPKM, correlation coefficient of three biological replicates for each sample was calculated. The correlation coefficients were $>0.94$ for almost all comparisons, suggesting that there was a perfect correlation among the biological replicates (Additional file 2: Figure S1).

\section{Whole-transcriptome identification and characterization of IncRNAs in melon}

A total of 124,979 unique transcripts were obtained from RNA-Seq data merged from all 18 samples. After seven sequential stringent filters (see materials and methods), 11,612 lncRNAs were identified, which were evenly distributed across 12 chromosomes in melon (Fig. 2). Among them, 11,122 lncRNAs were accumulated in both M1 and B29, and only 254 and 236 unique lncRNAs were specifically expressed in M1 and B29, respectively (Fig. 3a). Based on their genomic location and orientation relative to the nearest protein coding genes, lncRNAs are classified into lincRNA, intronic lncRNA and antisense lncRNA. Approximately $83.28 \%$ lncRNAs belonged to lincRNAs, $10.28 \%$ lncRNAs belonged to antisense lncRNA, and $6.44 \%$ lncRNAs were classified into intronic lncRNA in melon (Fig. 3b). The length and exon number of melon lncRNAs were analyzed compared with protein-coding transcripts for their characterization. As shown in Fig. 3c, the length of most lncRNAs $(\sim 68 \%)$ ranged within 200-300 nucleotides, whereas the length of most protein-coding transcripts mainly ranged in the size of $>1000$ nucleotides in melon. In addition, majority lncRNAs (90\%) contained one or two exons, while the number of exons for proteincoding genes ranged from one to $\geq 10$ (Fig. 3d). These results indicated that the majority of melon lncRNAs were relatively shorter in length and contained fewer exons compare to protein-coding transcripts.

\section{Differential expression of IncRNAs in response to PM infection}

To identify PM-responsive lncRNAs, their differential expressions were evaluated between PM infected samples and mock samples for both PM-resistant and PM-susceptible melons. The lncRNAs expressed with $\mid \log _{2}$ fold change $\mid \geq 1$ and adjusted $P$-values $<0.01$ were designated as DELs. More DELs were identified in PMresistant melon compared to PM-susceptible melon, while the number of down-regulated DELs was greater than that of up-regulated DELs in all comparison groups. As a result, a total of 117, 84, 105, 141 lncRNAs were found to be significantly up-regulated in B24, B48, M24, M48, respectively. Furthermore, a total of 205, 176, 224, 290 lncRNAs were found to be significantly down-regulated in B24, B48, M24, M48, respectively (Fig. 4a). Additionally, a total of 183 nd 387 lncRNAs were specifically differentially expressed in PMsusceptible melon and PM-resistant melon, respectively (Fig. 4b). The differential expression levels of eight highly altered DELs were experimentally validated by qRT-PCR. The results showed that the expression of LNC_010059, LNC_018602, LNC_023803 were significantly up-regulated at 24 and 48 hpi in PM-resistant melon after PM infection. However, the expression levels of these three lncRNAs were not changed in PMsusceptible melon (Fig. 5). Moreover, qRT-PCR analysis confirmed that the accumulation of LNC_000705, LNC_ 


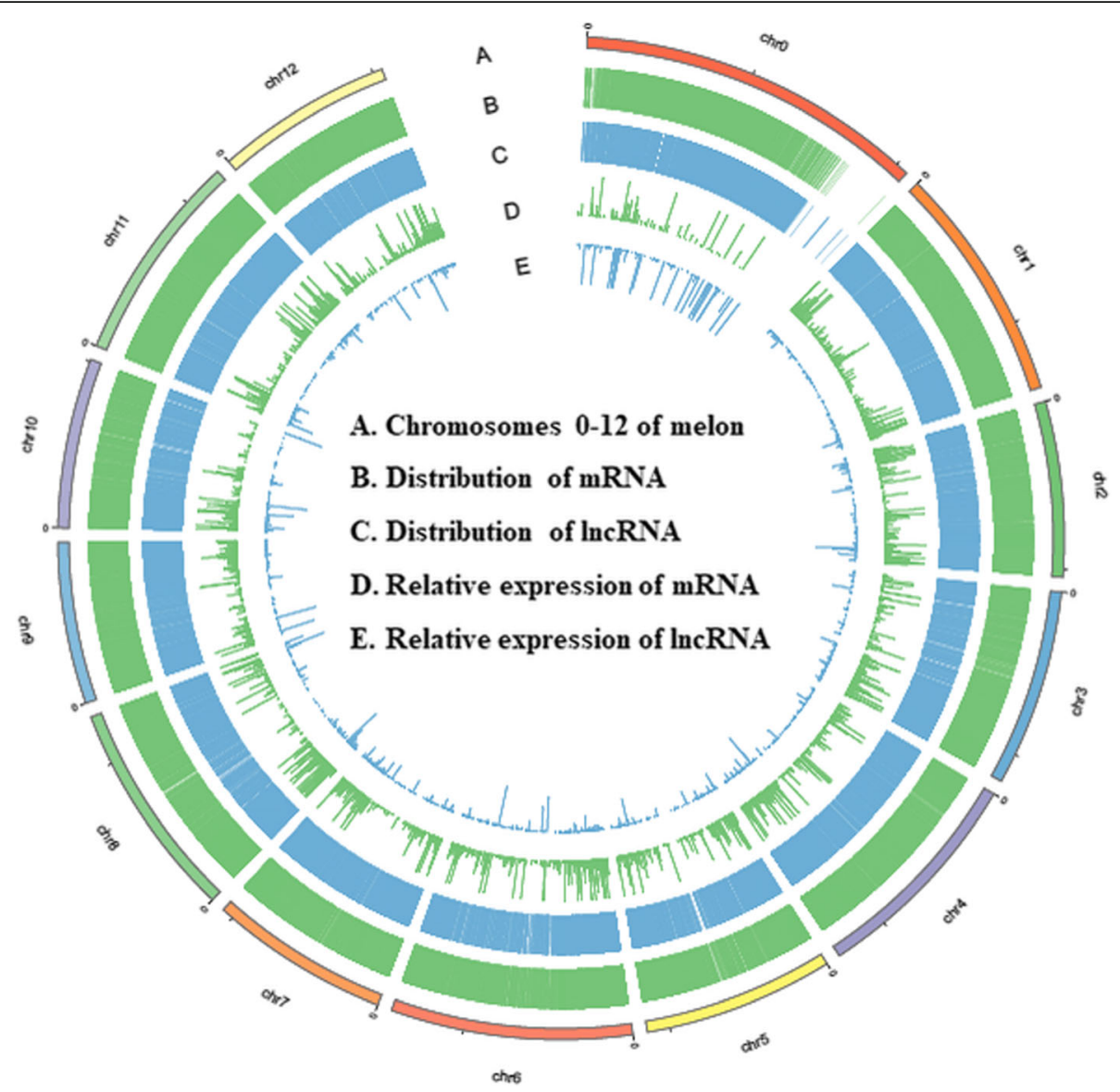

Fig. 2 Genome-wide distribution and expression of melon IncRNAs compared to that of protein-coding mRNAs. The expression level of IncRNAs and protein-coding mRNAs is presented as $\log _{10}$ FPKM

006883, LNC_009456, LNC_018800, LNC_019333 in PM-resistant melon were highly induced than that in PM-susceptible melon after PM infection, which were consistent with the RNA-seq results (Fig. 5), suggesting that the high throughput data were reliable.

\section{Target prediction and functional characterization of differentially expressed IncRNAs}

Generally, lncRNAs function in controlling the expression of their cis- or trans-target genes by forming lncRNA-target duplexes. In order to reveal the potential functions and regulatory mechanism of lncRNAs in response to PM infection, we characterized the target genes that were located $<10 \mathrm{~kb}$ from the DELs and analyzed their Gene Ontology (GO) terms. A total of 1232 protein-coding genes were predicted as target genes for all DELs, and these target genes were mainly enriched in three main GO categories, such as cellular component, molecular function and biological process (Fig. 6). The most abundant GO terms in the biological process were cell activation involved in immune response (GO:
0002263), metabolic process (GO: 0006629, lipid metabolic process), oxidation-reduction process (GO: 0004601, peroxidase activity; GO: 0045454, cell redox homeostasis) (Additional file 3: Figure S2). In addition, the molecular functions of these target genes were mainly enriched in "catalytic activity" and "oxidoreductase activity" (Fig. 6). The enrichment result suggested that the differentially expressed lncRNAs after PM infection may regulate the protein-coding genes involved in several important biological processes to resisting to PM infection.

\section{Identification of PM-resistant genes and expression analysis after PM infection}

With further analysis of the target genes of 387 DELs that were specific to PM-resistant melon, it was found that 532 protein-coding genes were co-located with DELs, and 440 and 335 protein-coding genes were positively co-expressed and negatively co-expressed with those DELs, respectively (Fig. 7a). Among those target genes, eight genes that might be directly involved in 


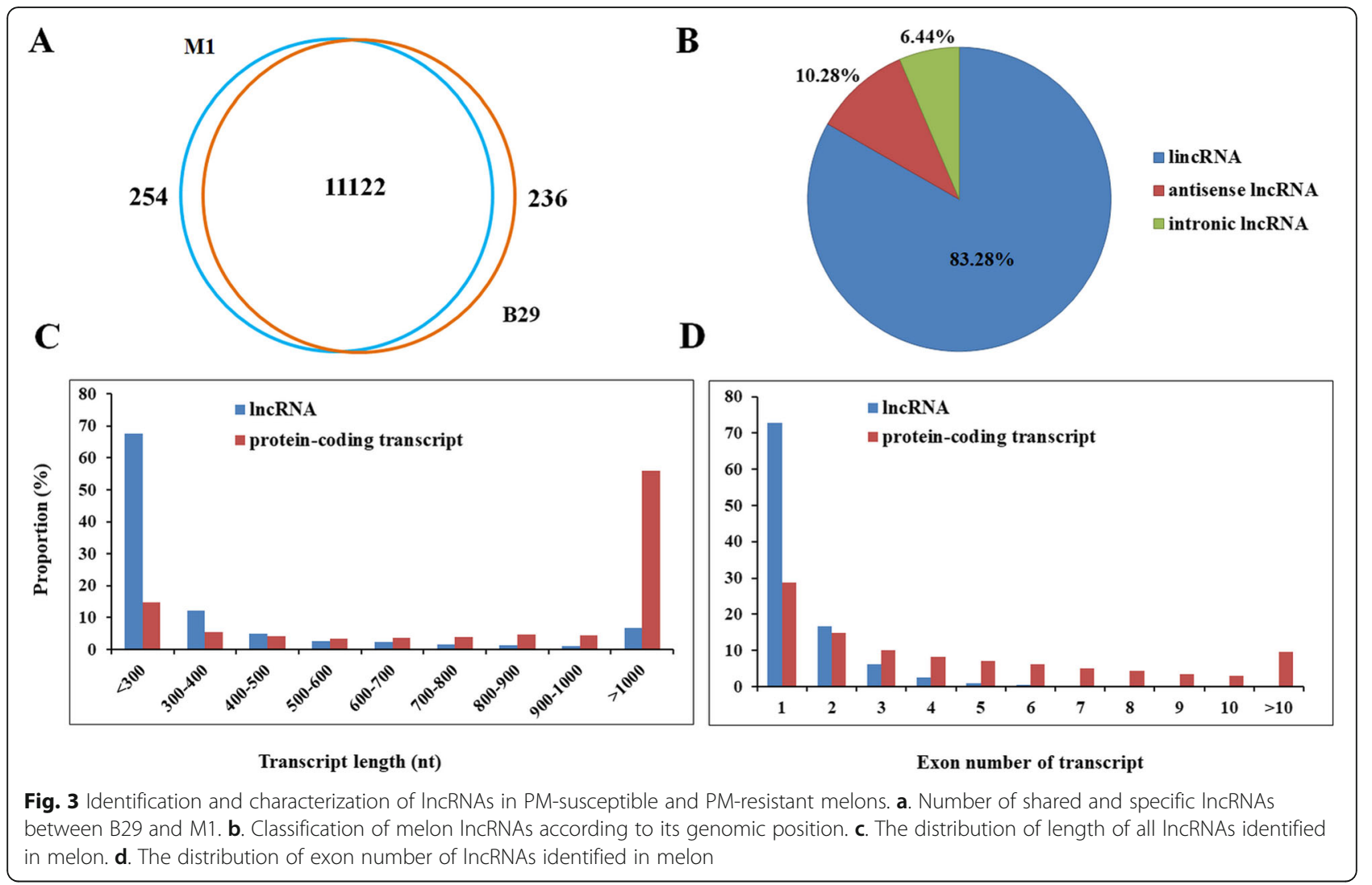

disease resistance were co-located with five DELs, and 30 genes that might be involved in PM resistance were co-expressed with 27 DELs (Table 1). MELO3C002814, encoding a LRR receptor-like kinase, was found to be located in the downstream 14,128 bp of LNC_010059 (Fig. 7b). Similarly, MELO3C014305, encoding a WRKY transcription factor, was found to be located in the upstream 10,972 bp of LNC_018800 (Fig. 7b). Furthermore, MELO3C015771, encoding a glutathione reductase, was co-expressed with LNC_018062 with a correlation coefficient of 0.96. To validate the putative expression patterns between DELs and their target genes, the expression levels of three DELs and their target genes after PM inoculation in both PM-susceptible and PMresistant melon were examined by qRT-PCR. It was found that CmWRKY21 and its paired IncRNA (LNC 018800), LNC_018062 and its paired target gene (MELO3C015771) exhibited a similar pattern in both PM-resistant melon and PM-susceptible melon, with up-regulated after PM infection in PM-resistant melon (Fig. 7c). Meanwhile, LNC_014937 and its paired target gene (CmMLO5) showed a similar decreased pattern in PM-resistant melons (Fig. 7c). In addition, the expression patterns of 38 PM-resistant genes are shown in a heatmap (Fig. 8). In particular, it was found that the accumulation levels of MELO3C023445, MELO3C006711, MELO3C017559, MELO3C024725 and MELO3C004323 in PM-resistant melon were much higher than that in PM-susceptible melon (Fig. 8). More importantly, these genes were significantly up- or down-regulated in PM-resistant melon at both 24 and $48 \mathrm{hpi}$ and no obvious differential expression of those genes was found in PMsusceptible melon after PM infection (Fig. 8). In addition, the expression of MELO3C012438 that encodes a Mildew Locus O (MLO) protein was decreased in PM-resistant melon after PM infection and no differential expression was observed in PMsusceptible melon.

\section{LncRNA act as precursors, targets or eTMs of miRNAs}

Numerous studies have reported that IncRNAs can interact with other ncRNAs such as miRNA to regulate various biological processes in many plants [23, 24]. On the one hand, many lncRNAs can act as potential miRNA precursors. On the other hand, IncRNAs could be targeted by miRNAs. In addition, plant IncRNAs could act as eTMs by binding to specific miRNA, competing with the target mRNA of miRNA and thus blocking the cleavage and alleviating the repression of its 


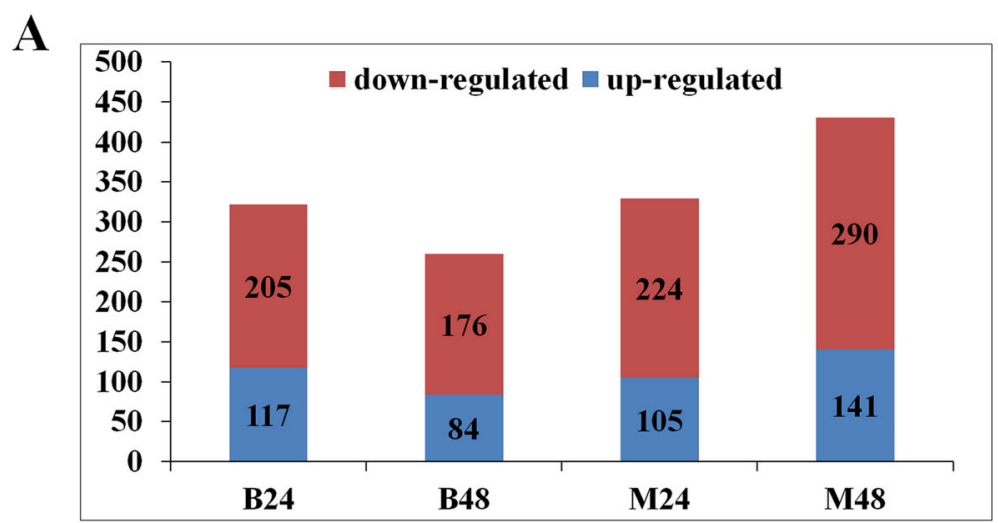

B

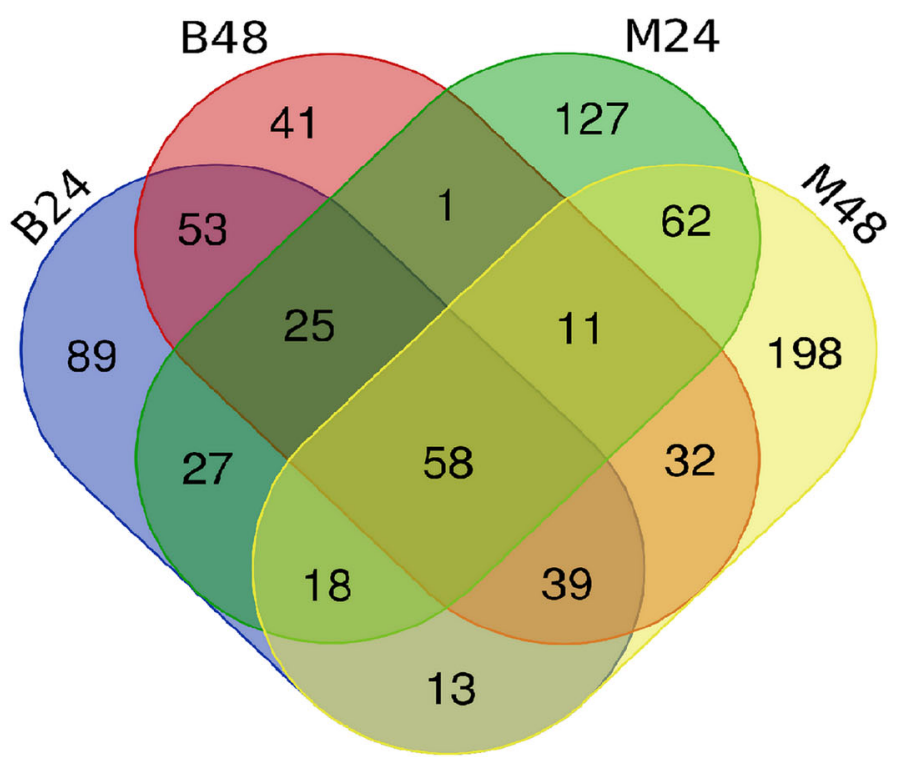

Fig. 4 Statistical analysis of DELs between PM-susceptible melon (B29) and PM-resistant melon (M1). a. Number of down- and up-regulated IncRNAs at 24 and 48 hpi compared with mock in B29 and M1. b. Number of shared and specific DELs in B29 and M1

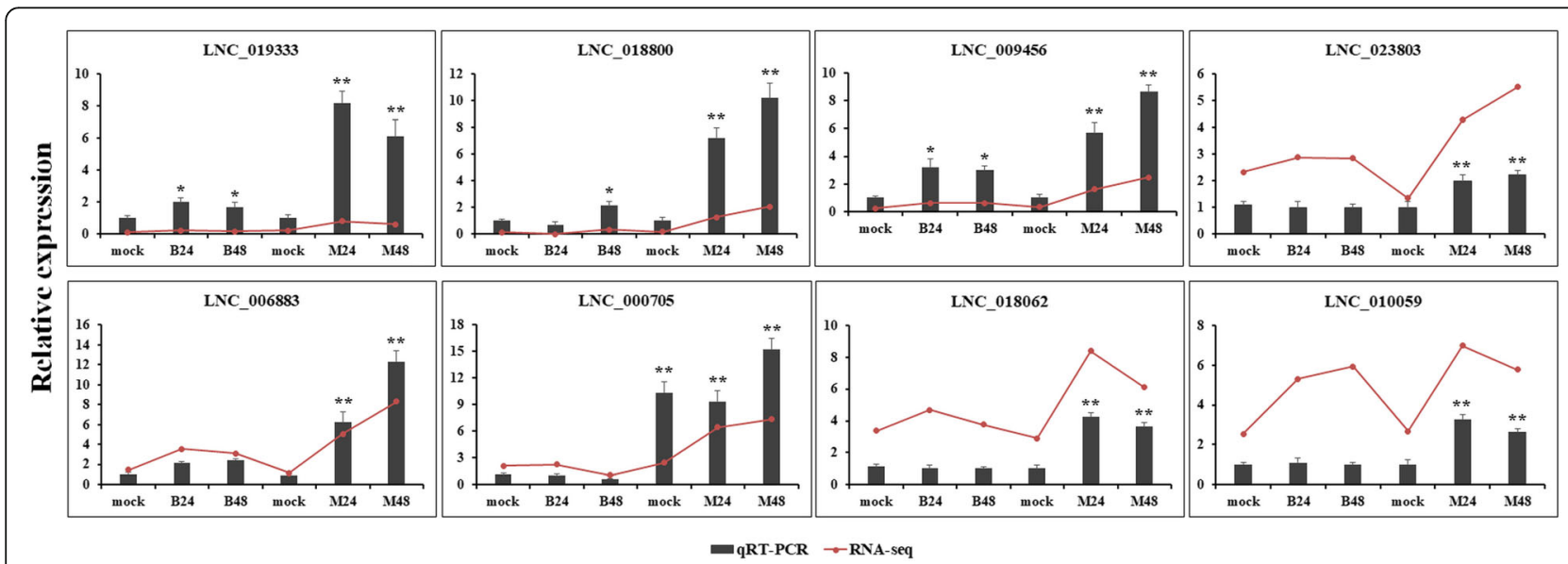

Fig. 5 Experimental validation of eight highly altered DELs by qRT-PCR. CmActin was used as internal reference. Relative level of IncRNAs was normalized to that in mock. The RNA-seq values were presented as log2 (FPKM value +1 ). Error bars indicate \pm SD of three biological replicates. Asterisks indicated a significant change $\left({ }^{*} P<0.05 ;{ }^{*} P<0.01\right)$ 


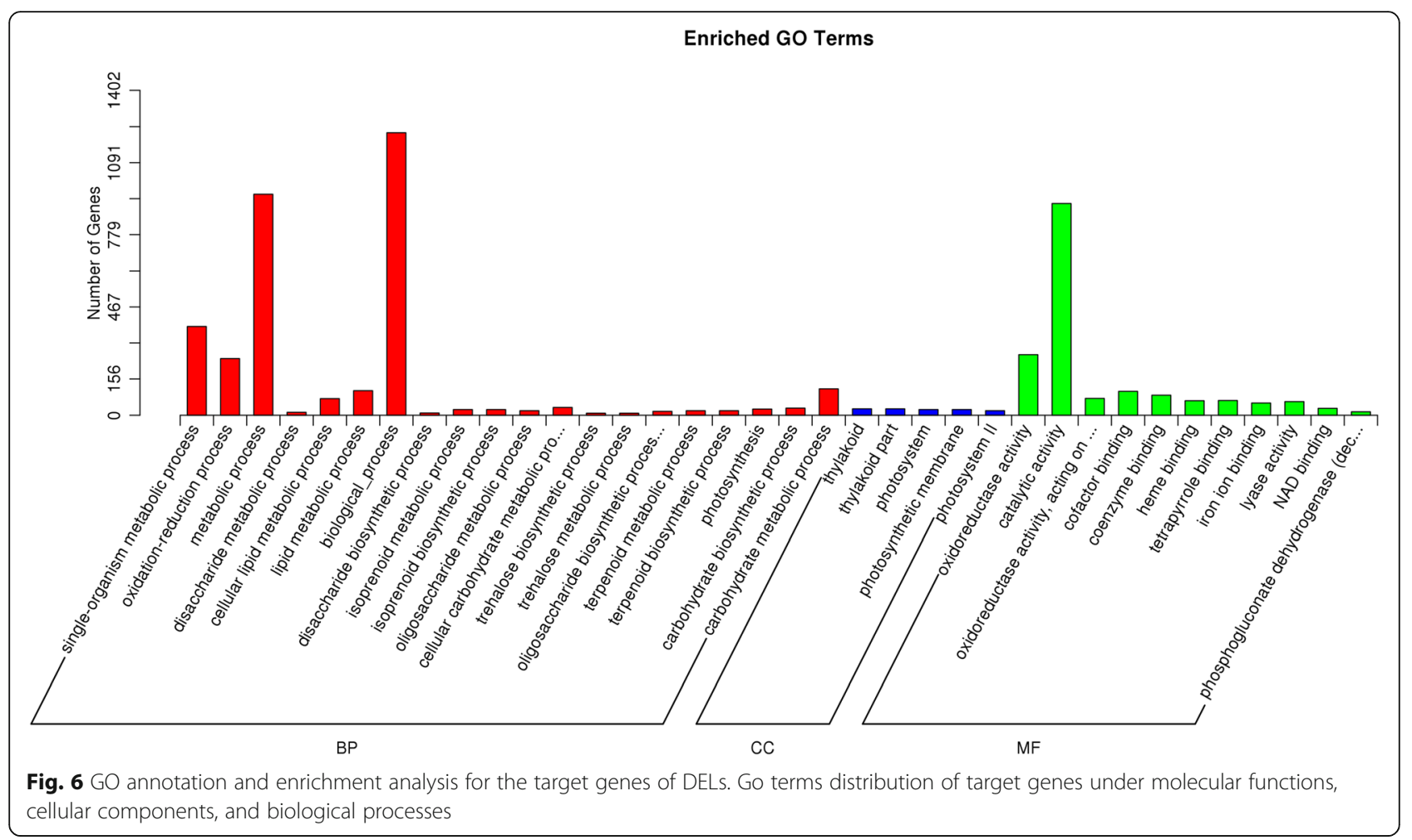

target gene [23]. In the present study, 23 lncRNAs were identified as the precursors of 19 miRNA families, including miR160, miR319, miR394, miR398 and miR408 that have been reported to play significant roles in mediating plant responses to phytopathogens (Table 2).
Meanwhile, 43 lncRNAs were predicted as the potential targets of 22 miRNA families and 13 lncRNAs as eTMs of 11 miRNAs (Table 3). For a fraction of miRNAs, only one target was identified, such as miR162, miR319, miR390 and others. However, most miRNAs were found

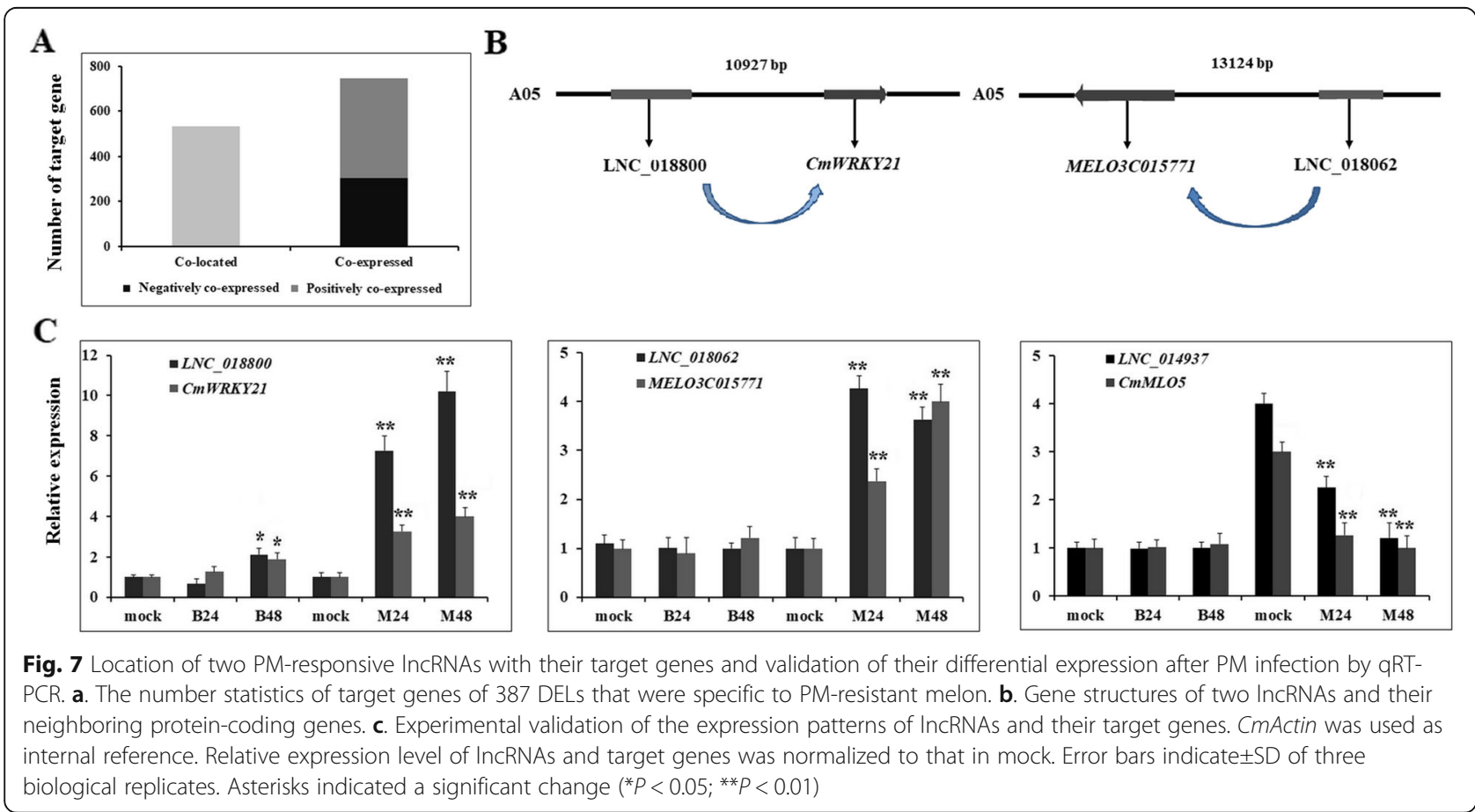


Table 1 Identification of target genes of DELs that related to diease resistance in melon

\begin{tabular}{|c|c|c|c|}
\hline LncRNA ID & Target gene ID & Functional annotation & Relationship \\
\hline LNC_010059 & MELO3C002814 & LRR receptor kinase & Co-located \\
\hline LNC_010059 & MELO3C002808 & GTPase-activating protein & Co-located \\
\hline LNC_000705 & MELO3C001005 & Cytochrome P450 & Co-located \\
\hline LNC_023803 & MELO3C017559 & Cytochrome P450 & Co-located \\
\hline LNC_019333 & MELO3C004321 & Disease resistance protein & Co-located \\
\hline LNC_019333 & MELO3C004323 & Disease resistance protein & Co-located \\
\hline LNC_019333 & MELO3C004324 & Disease resistance protein & Co-located \\
\hline LNC_018800 & MELO3C014305 & WRKY21 & Co-located \\
\hline LNC_009456 & MELO3C024725 & Disease resistance protein & Co-expressed \\
\hline LNC_006883 & MELO3C012016 & MLP-like protein & Co-expressed \\
\hline LNC_006883 & MELO3C015337 & NADPH-dependent reductase & Co-expressed \\
\hline LNC_018062 & MELO3C015771 & Glutathione reductase & Co-expressed \\
\hline LNC_008020 & MELO3C014655 & Peroxidase & Co-expressed \\
\hline LNC_018763 & MELO3C019440 & Ferredoxin & Co-expressed \\
\hline LNC_016838 & MELO3C023445 & LRR domain protein & Co-expressed \\
\hline LNC_014937 & MELO3C012438 & MLO & Co-expressed \\
\hline LNC_012950 & MELO3C021552 & Universal stress protein & Co-expressed \\
\hline LNC_003246 & MELO3C016714 & Protochlorophyllide reductase & Co-expressed \\
\hline LNC_009567 & MELO3C019735 & $\mathrm{ACO}$ & Co-expressed \\
\hline LNC_020345 & MELO3C016536 & NAC & Co-expressed \\
\hline LNC_006685 & MELO3C013917 & ERF5 & Co-expressed \\
\hline LNC_016838 & MELO3C014507 & F-box protein & Co-expressed \\
\hline LNC_003521 & MELO3C005466 & ERF & Co-expressed \\
\hline LNC_017611 & MELO3C009329 & Peroxidase & Co-expressed \\
\hline LNC_021129 & MELO3C026930 & ABA receptor & Co-expressed \\
\hline LNC_018663 & MELO3C014638 & Lipoxygenase & Co-expressed \\
\hline LNC_008020 & MELO3C005373 & F-box protein & Co-expressed \\
\hline LNC_006033 & MELO3C015186 & ferredoxin & Co-expressed \\
\hline LNC_012422 & MELO3C002203 & aldehyde dehydrogenase & Co-expressed \\
\hline LNC_025657 & MELO3C025034 & Peroxiredoxin & Co-expressed \\
\hline LNC_003761 & MELO3C003119 & Heat shock protein & Co-expressed \\
\hline LNC_011956 & MELO3C006711 & Universal stress protein & Co-expressed \\
\hline LNC_002899 & MELO3C002192 & Cytochrome P450 & Co-expressed \\
\hline LNC_011592 & MELO3C026489 & Cytochrome P450 & Co-expressed \\
\hline LNC_011422 & MELO3C000208 & Heat shock protein & Co-expressed \\
\hline LNC_010270 & MELO3C002228 & MYB1R1 & Co-expressed \\
\hline LNC_025117 & MELO3C023694 & Pathogen-related protein & Co-expressed \\
\hline LNC_000319 & MELO3C027077 & Heat shock protein & Co-expressed \\
\hline
\end{tabular}

to have more than one target genes. For example, six lncRNAs and seven IncRNAs were targeted by miR156 and miR7129, respectively. Among these miRNA targets, LNC_014183 was the target of miR398 that was recently confirmed to be involved in plant immunity.

\section{Discussion}

Emerging evidences showed that lncRNAs play significant roles in multiple biological processes, such as plant growth and development, fruit ripening, drought and salt stress response [13, 25]. In particular, lncRNAs have also been 


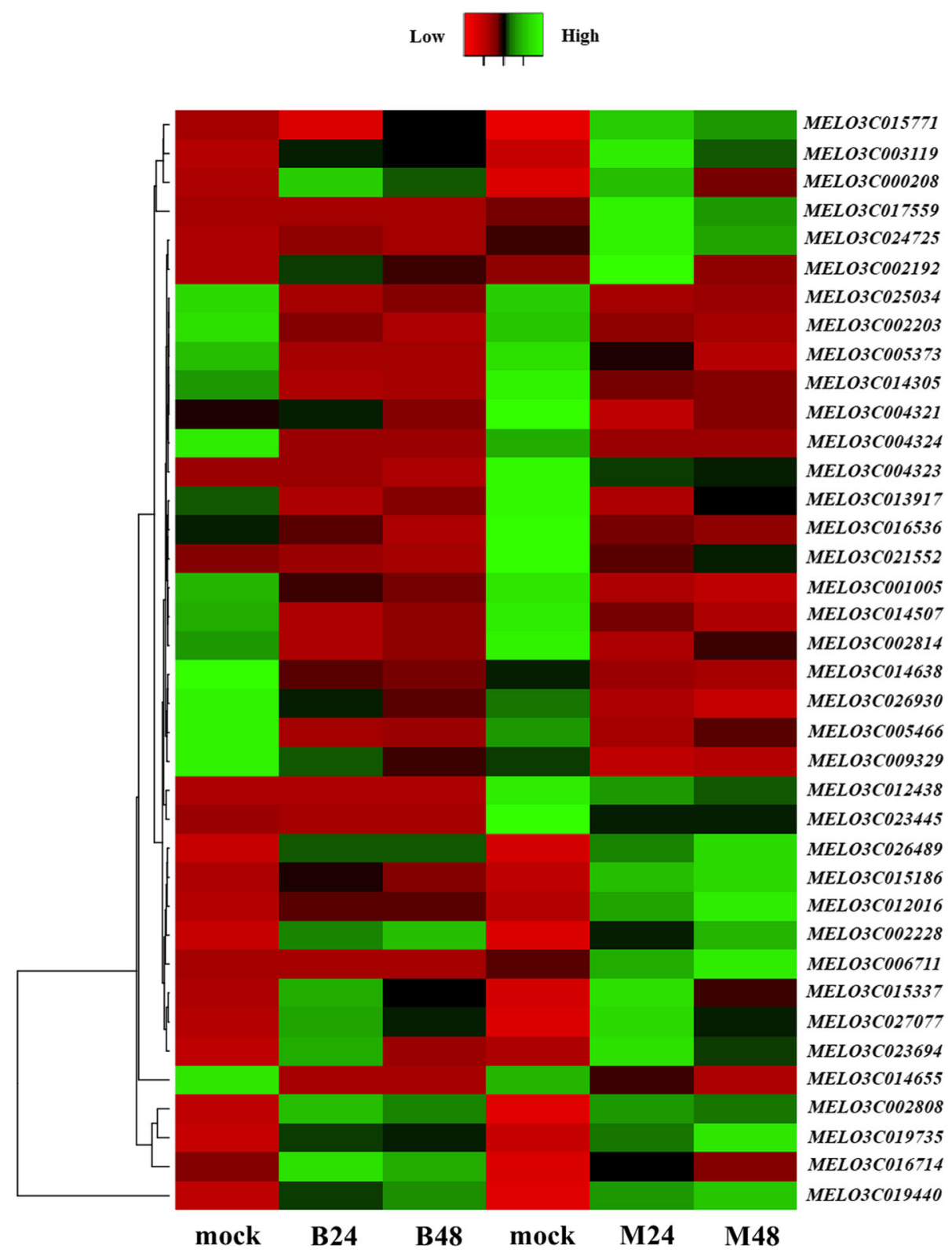

Fig. 8 Differential expression patterns of disease-related target genes of DELs after PM infection. The expression values were measured as fragments per kilobase of exon model per million mapped reads (FPKM) and presented as log2 (value +1$)$. High expression levels are shown in green and low expression levels are shown in red

shown to be involved in the resistance against multiple diseases in several plants [26, 27]. However, there is limited information about the roles of lncRNAs and their regulatory mechanism in resisting to PM infection in plants. Recently, the availability of melon genome sequences provided reference information for non-coding regions annotation and their functional analysis. In the present study, high-throughput RNA-seq and comparative transcriptome analysis were performed to identify PMresponsive lncRNAs from the leaves of PM-resistant and
PM-susceptible melons. In total, 11,612 lncRNAs were finally discovered. The number of hc-lncRNAs in melon was higher than that in Arabidopsis (6480), rice (2965), tomato (3679) and Chinese cabbage (4594), which can be attributed to the number of samples used for sequencing in our study were larger than that of other species. Furthermore, it was observed that lncRNAs in melon were relatively shorter in length and contained fewer exons compare to protein-coding transcripts, which was consistent with the results from all other plants $[8,11]$. 
Table 2 LncRNAs acting as miRNA precusors in melon

\begin{tabular}{|c|c|c|c|c|c|c|}
\hline IncRNA ID & IncRNA start & IncRNA end & miRNA ID & pre-miRNA length & Identity & E-value \\
\hline LNC_013051 & 236 & 417 & Cm-miR156 & 182 & 97 & $2.00 \mathrm{E}-27$ \\
\hline LNC_011277 & 323 & 532 & Cm-miR159 & 210 & 95 & $4.00 \mathrm{E}-28$ \\
\hline LNC_006685 & 464 & 562 & Cm-miR160 & 99 & 93 & $3.00 E-31$ \\
\hline LNC_012140 & 515 & 617 & Cm-miR162 & 103 & 95 & $2.00 \mathrm{E}-32$ \\
\hline LNC_026045 & 112 & 202 & Cm-miR164a & 91 & 100 & $2.00 \mathrm{E}-35$ \\
\hline LNC_014811 & 593 & 681 & Cm-miR164b & 89 & 95 & $4.00 \mathrm{E}-30$ \\
\hline LNC_001873 & 220 & 369 & Cm-miR166 & 150 & 97 & $3.00 \mathrm{E}-31$ \\
\hline LNC_004282 & 359 & 518 & Cm-miR167a & 160 & 94 & 4.00E-29 \\
\hline LNC_020891 & 51 & 156 & Cm-miR167c & 106 & 93 & $2.00 \mathrm{E}-29$ \\
\hline LNC_010843 & 105 & 308 & Cm-miR167d & 204 & 92 & $2.00 \mathrm{E}-28$ \\
\hline LNC_017947 & 119 & 235 & Cm-miR167e & 117 & 93 & $4.00 \mathrm{E}-32$ \\
\hline LNC_023691 & 106 & 315 & Cm-miR168 & 210 & 92 & $4.00 \mathrm{E}-30$ \\
\hline LNC_007808 & 245 & 380 & Cm-miR171 & 136 & 97 & $1.00 \mathrm{E}-28$ \\
\hline LNC_000014 & 497 & 602 & Cm-miR172 & 106 & 95 & $1.00 \mathrm{E}-30$ \\
\hline LNC_007125 & 236 & 475 & Cm-miR319 & 240 & 91 & $4.00 \mathrm{E}-30$ \\
\hline LNC_022969 & 192 & 337 & Cm-miR394 & 146 & 94 & $2.00 \mathrm{E}-30$ \\
\hline LNC_006844 & 55 & 143 & Cm-miR395 & 89 & 97 & 7.00E-34 \\
\hline LNC_002209 & 60 & 182 & Cm-miR396a & 123 & 96 & $1.00 \mathrm{E}-29$ \\
\hline LNC_011916 & 132 & 282 & Cm-miR396b & 151 & 95 & $1.00 \mathrm{E}-31$ \\
\hline LNC_010188 & 173 & 265 & Cm-miR397 & 93 & 95 & $2.00 \mathrm{E}-36$ \\
\hline LNC_011679 & 115 & 214 & Cm-miR398 & 100 & 100 & $2.00 \mathrm{E}-29$ \\
\hline LNC_006969 & 1228 & 1361 & Cm-miR408 & 134 & 97 & 4.00E-29 \\
\hline LNC_020158 & 73 & 181 & Cm-miR477 & 109 & 97 & $7.00 \mathrm{E}-26$ \\
\hline LNC_007291 & 157 & 320 & Cm-miR858 & 164 & 90 & $1.00 \mathrm{E}-28$ \\
\hline
\end{tabular}

Differential expression analysis revealed that a large number of lncRNAs were significantly differentially expressed after PM infection in both PM-resistant and PM-susceptible melons, which clearly suggested that these PM-responsive lncRNAs might be important regulators in PM resistance. A total of 183 and 387 lncRNAs were specifically differentially expressed in PM-susceptible melon and PM-resistant melon, respectively. More importantly, qRT-PCR analysis confirmed that the fold change of various lncRNAs in PM-resistant melon were larger than that in PM-susceptible melon, implying that a distinct disease response and function might exist in both melons. Thus, further functional study will be focused on these 387 DELs that were likely to play significant roles in resisting to PM, which contribute to the resistance phenotype in PM-resistant melon. Among 387 DELs, many lncRNAs such as LNC 019333, LNC_018800, LNC_023803, LNC_018062, LNC_010059 were obviously induced at both 24 and 48 hpi compared to mock samples after PM infection in PM-resistant melon, whereas these lncRNAs showed no differential expression either at 24 or at 48 hpi in PM- susceptible melon, which further confirmed that these lncRNAs might play vital roles in the biological processes that against to PM infection.

Previous studies have reported that the interaction between lncRNAs and their targeted mRNAs was one of the most important functional patterns of lncRNAs, and lncRNAs mainly function in regulating the expression of their neighboring genes either in cis or in trans manners $[6,28]$. To understand the regulatory pathways of lncRNAs in gene expression during PM infection, potential target genes of the DELs were predicted. Functional annotation results showed that a large number of target genes of lncRNAs encoded proteins that were involved in redox processes such as cytochrome $\mathrm{P} 450$, glutathione reductase and peroxidase. It has been proposed that glutathione reductase plays important roles in ROS scavenging pathway to prevent oxidative damage, which alleviates cell membrane injury after pathogen infection. Recently, it has been demonstrated that overexpression of glutathione reductase gene (SlGRE21) in tomato reduced ROS accumulation and enhanced the resistance against $P$. infestans [29]. Meanwhile, eight target genes, 
Table 3 LncRNAs that were predicted as miRNA targets and eTMs in melon

\begin{tabular}{|c|c|c|c|}
\hline LncRNA ID & miRNA ID & miRNA sequence & LncRNA sequence \\
\hline \multicolumn{4}{|c|}{ Targets } \\
\hline LNC_012115 & Cm-miR156 & ACTGTCTTCTATCTCTCGTG & TGACAGAAGAGAGTGAGCAC \\
\hline LNC_011287 & Cm-miR156 & TTCTGTCTTCTATCTCTGTT & TTGACAGAAGATAGAGAGCAC \\
\hline LNC_011878 & Cm-miR156 & СACTGTCTTCTCTCACTCGTG & TTGACAGAAGATAGAGAGCAC \\
\hline LNC_012070 & Cm-miR156 & AACCGTCTTCTGTTTCTCGTT & TTGACAGAAGATAGAGAGCAC \\
\hline LNC_013946 & Cm-miR156 & AACTGTCTTCTCTCACTCGTG & TTGACAGAAGATAGAGAGCAC \\
\hline LNC_021671 & Cm-miR156 & ACTITCTTCTATTITTCGTT & TGACAGAAGATAGAGAGCAC \\
\hline LNC_013934 & Cm-miR159 & AAACTTATCTTCCCTCGAGT & TाTGGATTGAAGGGAGCTCTA \\
\hline LNC_027695 & Cm-miR159 & TAACCTGACTTCCCTCGAGGA & TITGGATTGAAGGGAGCTCTA \\
\hline LNC_006537 & Cm-miR162 & AGCTATTTCGATACGTAGTC & TCGATAAACCTCTGCATCCAG \\
\hline LNC_016936 & Cm-miR164 & ACCTCTACGTCTCGTGTACTC & TGGAGAGGCAGGGCACATGCT \\
\hline LNC_017766 & Cm-miR164 & ACTTCTCCGTCCCGTATTCGA & TGGAGAGGCAGGGCACATGCT \\
\hline LNC_020709 & Cm-miR164 & ACTTCTCCGTTACGTGTAGA & TGGAGAGGCAGGGCACATGCT \\
\hline LNC_000218 & Cm-miR166 & AGCCTGGTCCGAAGTAAGGGG & TCGGACCAGGCTTCATTCCCC \\
\hline LNC_008758 & Cm-miR166 & AGCCTGGTCCGAAGTAAGGAG & TCGGACCAGGCTTCATTCCCC \\
\hline LNC_018819 & Cm-miR167 & AGTTCGACGGTCGTACTAGAT & TGAAGCTGCCAGCATGATCTA \\
\hline LNC_021730 & Cm-miR167 & GCTTCGACTGTCGTACTGTT & TGAAGCTGCCAGCATGATCTA \\
\hline LNC_022241 & Cm-miR168 & TCGCTTGGTGCAGGTCGGGA & AGCGAACCACGTCCAGCCCT \\
\hline LNC_004566 & Cm-miR169 & TTCGTTTCCTACTTAACCAAC & AAGCCAAGGATGAATTGCCGG \\
\hline LNC_010303 & Cm-miR169 & ATCGGTTCCTGCTGAACGGCG & AAGCCAAGGATGAATTGCCGG \\
\hline LNC_022185 & Cm-miR1863 & AGCTCTGATACCATGTTAGATTTG & ACGAGACTATGGTATAATTTGAC \\
\hline LNC_027695 & Cm-miR319 & TTGGACTGAAGGGAGCTCCC & AACCTGACTTCCCTCGAGGA \\
\hline LNC_008308 & Cm-miR390 & AAGCTCAGGAGGGATAGCGCC & GTCGAGTTCTCCCTATCTGT \\
\hline LNC_005952 & Cm-miR393 & TCCAAAGGGATCGCATTGATC & AGGGTTACCTAGCGTAACTAC \\
\hline LNC_014927 & Cm-miR394 & TTGGCATTCTGTCCACCTCC & AACCGTAAGACAGGTGGAGG \\
\hline LNC_024182 & Cm-miR395 & CTGAAGTGTTTGGGGGAACTC & TACTTCACAAACTCCCTTAT \\
\hline LNC_006804 & Cm-miR398 & TGTGTTCTCAGGTCGCCCCTG & ACACAAGAGTCCAGTGGGGAA \\
\hline LNC_014183 & Cm-miR398 & TGTGTTCTCAGGTCGCCCCTG & CCATAAGAGTCCGGCGG-AAC \\
\hline LNC_020691 & Cm-miR399 & TGCCAAAGGAGAATTGCAC & TCTGTTCCTCTTAACGG \\
\hline LNC_022982 & Cm-miR399 & TGCCAAAGGAGAATTGCAC & ACAGTTCCTCTTAACGTA \\
\hline LNC_005545 & Cm-miR477 & СTCTCCCTCAAAGGCTTCTG & GGGTGGGAGTTCCGAAAG \\
\hline LNC_007655 & Cm-miR530 & TGCATTTGCACCTGCACCTT & ATGTAAACGTGGATGTAGACA \\
\hline LNC_004049 & Cm-miR854 & GATGAGGATAGTGAGGAGGAG & СТАСТССТАТСАСТССТССТС \\
\hline LNC_012258 & Cm-miR854 & GATGAGGATAGTGAGGAGGAG & СТАССССТАТСАСТАСТССТС \\
\hline LNC_020802 & Cm-miR854 & GATGAGGATAGTGAGGAGGAG & TCAGTCCTATCACTCCTTCTC \\
\hline LNC_026553 & Cm-miR854 & GATGAGGATAGTGAGGAGGAG & СТТСТССТАТСАСТССТССТС \\
\hline LNC_011348 & Cm-miR858 & TCTCGTTGTCTGTTCGACCTT & AGGGCAACAGGCAAGCTTGTT \\
\hline LNC_007955 & Cm-miR7129 & AGTCAAATCTAAACGATCGTGTAT & ACAGTTAGATTTGCTAGCAAATA \\
\hline LNC_008970 & Cm-miR7129 & AGTCAAATCTAAACGATCGTGTAT & TAAGTTAGATTTGCTAGCACATG \\
\hline LNC_009809 & Cm-miR7129 & AGTCAAATCTAAACGATCGTGTAT & TTGGTTTAGATTTGCTGACACATG \\
\hline LNC_010638 & Cm-miR7129 & AGTCAAATCTAAACGATCGTGTAT & CGGTTTAGATTTGCTAGAACATT \\
\hline LNC_016701 & Cm-miR7129 & AGTCAAATCTAAACGATCGTGTAT & TTAGTTAGATTTGCTAGCACATG \\
\hline LNC_023506 & Cm-miR7129 & AGTCAAATCTAAACGATCGTGTAT & TCAGTTTAGATTTGTTAGCACATG \\
\hline
\end{tabular}


Table 3 LnCRNAs that were predicted as miRNA targets and eTMs in melon (Continued)

\begin{tabular}{|c|c|c|c|}
\hline LncRNA ID & miRNA ID & miRNA sequence & LncRNA sequence \\
\hline LNC_024789 & Cm-miR7129 & AGTCAAATCTAAACGATCGTGTAT & GAAGTTAGATTGCTAGCACGA \\
\hline LNC_021385 & Cm-miR7130 & GTTGGAATGTGCGAGATGTGTGC & TCAACTITACACGCTCTACACACG \\
\hline \multicolumn{4}{|l|}{ eTMs } \\
\hline LNC_015061 & Cm-miR156 & TGACAGAAGA---GAGTGAGCAC & ACTGTCTTCTAAGTTCACACGTG \\
\hline LNC_001794 & Cm-miR159 & ATTGGATTGA---AGGGAGCTCCT & TAACCTAACTATGTTCCTCGAGTA \\
\hline LNC_005497 & Cm-miR167a & TGAAGCTGCC---AGCATGATCTT & ACTTCGACGGCTITTGTACAAGGA \\
\hline LNC_004707 & Cm-miR167b & TGAAGCTGC---CAGCATGATCTG & ACTTCGACGACTGTCGTGATAGGC \\
\hline LNC_001168 & Cm-miR169 & GAGCCAAGAA---TGACTTGCCGG & CTCGGTTCTTGAAACTGAACGGCT \\
\hline LNC_027362 & Cm-miR172 & AGAATCTTGA---TGATGCTGCAT & TATTAGAACTACTACTATGACGCA \\
\hline LNC_017301 & Cm-miR394 & TTGGCATTC---TGTCCACCTCC & AACCGTAAGTCCATAGGTGAAGT \\
\hline LNC_013466 & Cm-miR395 & TTGAAGTGTT---TGGGGGAACTC & AACTTCACAAAACACTCACTTGAG \\
\hline LNC_005015 & Cm-miR398 & TGTGTTCTC---AGGTCACCCCTT & ACACAAGAGAACTCCAGTCGAGAA \\
\hline LNC_019732 & Cm-miR477 & СTCTCCCTC---AAAGGCTTCTG & GAGAGGGAGAGGTITTCTAAAAC \\
\hline LNC_023016 & Cm-miR854 & GATGAGGATA---GTGAGGAGGAG & СТACTCCTATTGCCACTCTTACTA \\
\hline LNC_027169 & Cm-miR854 & GATGAGGATA---GTGAGGAGGAG & СTACTCCTACGCTCACTACTACTT \\
\hline LNC_027417 & Cm-miR854 & GATGAGGATA---GTGAGGAGGAG & СTACTCCTATTAAAAGTCCTGCTC \\
\hline
\end{tabular}

encoding for disease resistance protein, were identified including pathogen-related gene, LRR receptor gene, and universal stress gene. Moreover, five genes encoding for transcription factors, such as WRKY, ERF, MYB and NAC, were also discovered. In the past decades, increasing studies have revealed that a large number of WRKY family transcription factors were involved in response to biotic defense (bacterial, fungal and viral pathogens) [30]. Subsequently, our qRT-PCR results verified that similar expression patterns were induced between $\mathrm{LNC}_{-}$ 018062 and MELO3C015771 (glutathione reductase coding gene), LNC_018800 and CmWRKY21 after PM infection, suggesting that lncRNAs might control the expression of CmWRKY21 and redox pathway genes, leading to the resistance to PM in melon.

Interestingly, a $M L O$ family gene that acts as susceptibility factor towards PM was found to be targeted by LNC_014937. It is speculated that plant-specific MLO proteins contain seven transmembrane domains, which are likely to modulate vesicle-associated defense responses at the cell periphery [31]. MLO resistance has been reported in barley, Arabidopsis, pea, cucumber, tomato and many other species. In tomato, the loss-offunction of $M L O$ family gene (SIMLO1) led to high resistance against PM [32]. Besides, in Arabidopsis thaliana, T-DNA insertion mutations in three $M L O$ homologs (AtMLO2, AtMLO6 and AtMLO12) contributed to significant PM resistance, although the mutation in AtMLO2 had a significant effect compared to mutations in other two genes [33]. These studies showed that MLO proteins in plants function as characteristic susceptibility genes (S-genes) and play a negative regulatory role in resisting to PM. In the present study, qRTPCR results revealed that the expression level of both LNC_014937 and CmMLO5 in PM-resistant melon was significantly higher than that in PM-susceptible melon. Meanwhile, the expression of both LNC_014937 and CmMLO5 was significantly down-regulated in PMresistant melon, although no differential expression was found in PM-susceptible melon, implying that the $\mathrm{LNC}_{-}$ 014937 and CmMLO5 module might play important roles in melon responses to PM. Subsequently, further molecular and biological experiments should be carried out to elucidate their biological function and regulatory mechanisms in resisting PM infection.

It has been reported that lncRNAs can control gene expression through various pathways. They can act as targets or eTMs of miRNA to restrict the cleavage of target mRNA mediated by miRNA, and thus activate target gene expression $[24,34]$. Similar to the results in Arabidopsis, rice, cotton and other plants, many melon lncRNAs were predicted to be miRNAs target or decoys. In the present study, 56 lncRNAs were predicted to be potential targets or eTMs of 32 family miRNAs including miR156, miR159, miR164, miR166, miR167, miR169 and others. Among them, several miRNAs, such as miR398, miR477, miR854 and miR858, have been proved to play important roles in response to various biotic and abiotic stresses. Recently, miR398 was found to be involved in immunity against the blast fungus through regulating two genes encoding $\mathrm{Cu} / \mathrm{Zn}$-superoxidase dismutase (CSD) [35]. Besides, miR858-mediated regulation 
of phenylpropanoid biosynthetic pathway also played important role in Arabidopsis immunity [36]. These results demonstrated that certain interactions between lncRNAs and miRNAs may exist in melon, which provides a solid foundation for further investigation in the function of lncRNAs in PM tolerance.

\section{Conclusions}

In this study, a total of 11,612 hc-lncRNAs were identified in melon. Further characterization analysis showed that IncRNAs in melon were distributed across all 12 melon chromosomes, and $>85 \%$ were from intergenic regions. Besides, lncRNAs in melon were relatively shorter in length and contained fewer exons compare to protein-coding genes. A total of 407 and 611 lncRNAs were found to be differentially expressed after PM infection in PM-susceptible and PM-resistant melons, respectively. Furthermore, 1232 putative targets of differentially expressed lncRNAs were discovered and functional annotation showed that a large number of target genes of lncRNAs encoded proteins that were involved in redox processes, such as cytochrome P450, glutathione reductase and peroxidase. Interestingly, a number of lncRNAs can act as potential miRNA precursors. Meanwhile, lncRNAs could also act as targets or eTMs of miRNAs. Collectively, our findings provide new insights into the role of lncRNAs for further study on the function and regulatory mechanisms of lncRNAs in PM resistance.

\section{Methods}

Plant materials, growth conditions and powdery mildew fungus inoculation

A highly resistant cultivated melon (M1) and a highly susceptible cultivated melon (B29) to powdery mildew fungus were selected as plant materials and grown in a greenhouse with a photoperiod of $16 / 8 \mathrm{~h}$ (day/night) at $28{ }^{\circ} \mathrm{C} / 20^{\circ} \mathrm{C}$ (day/night). $\mathrm{M} 1$ is an inbred line that was self-pollinated for thirteen generations, with thick rind and high net density. B29 is an inbred line separated from a commercial cultivar, with a thin and smooth rind (no netting). The genetic background of these two lines has been highly stable. Powdery mildew fungus was collected from cultivated melon grown in the experimental farm of Shandong Academy of Agricultural Sciences with normal day/night period. Plants with two or three true leaves were inoculated by powdery mildew fungus at a concentration of $1 \times 10^{6} / \mathrm{mL}$ as previously described [37]. Control samples were treated with water (mock). Leaves of both M1 and B29 were harvested at 24 and 48 $\mathrm{h}$ post inoculation (named as M24, M48, B24, B48, respectively), and immediately frozen in liquid nitrogen and stored at $-80^{\circ} \mathrm{C}$ for the following RNA extraction.
Three biological replicates were prepared for each sample.

\section{Total RNA extraction, library construction and paired-end strand-specific sequencing}

Total RNAs were extracted from all samples using Trizol reagent following the manufacturer's instructions (Invitrogen, CA, USA) and the integrity was examined on $1 \%$ agarose gel. RNA concentration and quality were measured by NanoPhotometer spectrophotometer (IMPLEN, CA, USA) and Bioanalyzer 2100 system (Agilent Technologies, CA, USA). A total amount of $3 \mu \mathrm{g}$ RNA per sample was used as input material for RNA sample preparation. Firstly, ribosomal RNA was removed using rRNA Removal Kit (Epicentre, USA), and rRNA free residue was purified by ethanol precipitation. Subsequently, sequencing libraries were generated using rRNA-depleted RNA by NEBNext Ultr Directional RNA Library Prep Kit for Illumina (NEB, USA) following manufacturer's instructions. After library generation, 150 bp paired-end reads were generated on an Illumina Hiseq 4000 platform. The raw sequence reads are available for download from the NCBI sequence read archive database (Accession number: SRR9129105-SRR9129122).

\section{RNA transcripts assemble and identification of IncRNAs}

To assemble full-length transcripts, the raw data were preprocessed by the Fastx-toolkit pipeline (http://hannonlab. cshl.edu/fastx_toolkit/) to trim the adapter sequences and remove low-quality sequences. All clean reads were aligned to melon reference genome (http://cucurbitgenomics.org/organism/18) using HISAT2 (v2.0.4; https:// ccb.jhu.edu/software/hisat2/index.shtml). Only reads with no more than two mismatches were used to generate fulllength transcripts of each sample separately using StringTie (version 1.3.1; http://ccb.jhu.edu/software/stringtie/). To identify lncRNA, all transcripts were firstly aligned to housekeeping ncRNA databases (version 1.2; http://bioinf. scri.sari.ac.uk/cgi-bin/plant_snorna/home) to exclude tRNAs, snRNAs and snoRNAs. Then, the remaining transcripts were compared with melon genome annotated protein sequences (http://cucurbitgenomics.org/organism/18) using BlastX. Non-redundant transcripts with significant alignment $(P<1.0 \mathrm{E}-5$, identity $>90 \%$, coverage $>80 \%)$ to melon proteins were excluded. Perl scripts were used to exclude transcripts shorter than 200 nucleotides and transcripts with a FPKM score higher than 1 in at least one sample. Finally, the remaining transcripts were uploaded to the Coding Potential Calculator (CPC), Coding-Non-Coding-Index (CNCI), Pfam Scan (Pfam-sca) and phylogenetic codon substitution frequency (phyloCSF) programs to test protein-coding potential. Transcripts predicted with coding potential by at least one of 
the four tools above were filtered out, and those without coding potential were identified as candidate lncRNAs.

\section{Quantification and differential expression analysis of IncRNAs}

Cuffdiff program (v2.1.1) was used to calculate FPKM values of lncRNAs in each sample [38]. FPKM means fragments per kilo-base of exon per million fragments mapped, which is calculated based on the length of the fragments and reads count mapped to this fragment. Furthermore, Cuffdiff program (v2.1.1) was also used to calculate adjusted $P$-values between two samples with three biological replicates. Then, differentially expressed lncRNAs were identified using a criterion of $\mid \log _{2}$ fold change $\mid \geq 1$ and adjusted $P$-values $<0.01$. The adjusted $P$-values were calculated using Benjamini-Hochberg procedure. Hierarchical clustering heat map was generated using pheatmap in $\mathrm{R}$ package (https://www.r-project. org/) according to the euclidean distance method.

Validation of differentially expressed IncRNAs by qRT-PCR To confirm the differential expression of lncRNAs, qRTPCR method was performed to assess the relative expression quantity of IncRNAs. Total RNAs were extracted from the leaf samples using Trizol reagent following the manufacturer's instructions (Invitrogen, CA, USA). First strand cDNA was synthesized from $3 \mu \mathrm{g}$ of RNA using random hexamer primer and M-MuLV Reverse Transcriptase. Second strand cDNA synthesis was performed using DNA Polymerase I and RNase H. SYBR Green Master Mix (Bio-Rad, Hercules, California) was used in all qRT-PCR reactions with an initial denaturing step of $95^{\circ} \mathrm{C}$ for $5 \mathrm{~min}$, followed by 40 cycles of $95^{\circ} \mathrm{C}$ for $20 \mathrm{~s}, 65^{\circ} \mathrm{C}$ for $20 \mathrm{~s}$ and $72^{\circ} \mathrm{C}$ for $20 \mathrm{~s}$ on an ABI 7500 Real Time PCR system (Applied Biosystems, Waltham, Massachusetts). Three biological replicates were prepared for each sample and Cmactin was used as internal reference gene. Relative expression was calculated using the $2^{-\Delta \Delta C t}$ method and all data were expressed as means \pm SDs from three independent experiments. Duncan's multiple range tests were used to determine the statistical difference between samples $(P<0.01)$. Primers used in all qRT-PCR experiments are listed in Additional file 4: Table S2.

\section{Identification of IncRNA targets and gene ontology enrichment analysis}

To identify the target genes of differentially expressed lncRNAs, a Perl script was used to identify cis target genes located $10 \mathrm{~kb}$ upstream or downstream of lncRNAs, and for the identification of antisense target genes that can interact with lncRNAs to produce RNA duplex, RNAplex tool was used to examine the RNA duplex formation by calculating minimum free energy
(MFE) based on their respective structures [39]. Gene Ontology (GO) enrichment analysis of lncRNA target genes was performed using GOseq $\mathrm{R}$ package [40]. GO terms with corrected $P$-value $<0.05$ were considered significantly enriched. We used the Benjamini-Hochberg Correction for the $p$-value to obtain a corrected $p$-value.

\section{Identification of IncRNAs that act as miRNA precursors, targets or eTMs}

LncRNAs that may act as miRNAs precursors were predicted by aligning all lncRNA sequences against the known miRNA precursor sequences in miRBase database using BLASTN with an identity $>90 \%$ and cutoff Evalue $<1.0 \mathrm{E}-5$. To obtain lincRNAs that can act as miRNA targets, psRNATarget was used with following rules: at most, one mismatch was allowed between the 9th and 12th positions of the $5^{\prime}$-end of miRNA sequences, the total number of mismatches in other regions were not allowed to exceed 4 , and no continuous mismatches were allowed [41]. The miRNA eTMs from all lncRNAs were predicted using psMimic software according to following rules: (1) bulges were only permitted at $5^{\prime}$-end ninth to 12 th positions of miRNA sequence; (2) the bulge in lncRNAs should be composed of only three nucleotides; and (3) total mismatches within lncRNA and miRNA pairing regions should be no more than three except for the central bulge [42].

\section{Supplementary information}

Supplementary information accompanies this paper at https://doi.org/10. 1186/s12864-020-6546-8.

Additional file 1: Table S1. Summary statistics of RNA-seq data and mapping result.

Additional file 2: Figure S1. The correlation coefficients among all 18 samples.

Additional file 3: Figure S2. The most enriched GO terms of IncRNA targets in biological process.

Additional file 4: Table S2. Oligonucleotide primer sequences used for qRT-PCR.

\begin{abstract}
Abbreviations
DEL: Differently expressed IncRNA; eTM: Endogenous target mimic; FPKM: Fragments per kilo-base of exon per million fragments; GO: Gene ontology; IncRNAs: Long non-coding RNAs; MFE: Minimum free energy; miRNA: microRNA; MLO: Mildew Locus O; PM: Powdery mildew; qRTPCR: quantitative RT-PCR; siRNA: Small interfering RNA
\end{abstract}

\section{Acknowledgements}

Not applicable.

\section{Authors' contributions}

CG designed the research and performed the bioinformatics analysis. JLS contributed to RNA extraction and qRT-PCR. YMD finalized the figures and tables. SHX managed the plant materials and LFM analyzed the data. ZGJ wrote the original manuscript and CQW revised the original manuscript. All authors have read and approved the final manuscript. 


\section{Funding}

This work was financially supported by grants from the Natural Science Foundation of Shandong Province (ZR2018LC017), the National Technical System of Watermelon and Melon Industry (CARS-25), the National Key Research and Development Program of China (2018YFD0100705), Shandong Provincial Key Research and Development Program (2019GNC106029) and Agricultural Science and Technology Innovation Project of Shandong Academy of Agricultural Sciences (CXGC2018E08). The funding bodies played no role in the design of the study and collection, analysis, and interpretation of data and in writing the manuscript.

\section{Availability of data and materials}

The raw sequence reads are available for download from the NCBI sequence read archive database (Accession number: SRR9129105-SRR9129122).

\section{Ethics approval and consent to participate}

Not applicable.

\section{Consent for publication}

Not applicable.

\section{Competing interests}

The authors declare that they have no competing interests.

\section{Author details}

'Shandong Key Laboratory of Greenhouse Vegetable Biology, Shandong Branch of National Improvement Center for Vegetable, Vegetable Science Observation and Experiment Station in Huang huai District of Ministry of Agriculture (Shandong), Institute of Vegetables and Flowers, Shandong Academy of Agricultural Sciences, Jinan 250100, China. ${ }^{2}$ College of horticulture, Jilin Agricultural University, Changchun 130118, China.

\section{Received: 22 September 2019 Accepted: 31 January 2020} Published online: 05 February 2020

\section{References}

1. Djebali S, Davis CA, Merkel A, Dobin A, Lassman T, Mortazavi A, et al. Landscape of transcription in human cells. Nature. 2012;489:101-8.

2. Ponting $\mathrm{CP}$, Oliver PL, Reik W. Evolution and functions of long noncoding RNAs. Cell. 2009;136:629-41.

3. Axtell MJ. Classification and comparison of small RNAs from plants. Annu Rev Plant Biol. 2013:64:137-59.

4. Mercer TR, Mattick JS. Structure and function of long noncoding RNAs in epigenetic regulation. Nat Struct Mol Biol. 2013:20:300.

5. Chen YA, Aravin AA. Non-coding RNAs in transcriptional regulation: the review for current molecular biology reports. Curr Mol Biol Rep. 2015;1:10-8.

6. Yamada M. Functions of long intergenic non-coding (linc) RNAs in plants. J Plant Res. 2017:130:67-73.

7. Wu H, Yang L, Chen LL. The diversity of long noncoding RNAs and their generation. Trends Genet. 2017;33:540-52.

8. Li L, Eichten SR, Shimizu R, Petsch K, Yeh CT, Wu W, et al. Genome-wide discovery and characterization of maize long non-coding RNAs. Genome Biol. 2014:15:R40

9. Wang H, Chung PJ, Liu J, Jang IC, Kean MJ, Xu J, Chua NH. Genome-wide identification of long noncoding natural antisense transcripts and their responses to light in Arabidopsis. Genome Res. 2014;24:444

10. Zhang YC, Liao JY, Li ZY, Yu Y, Zhang JP, Li QF, et al. Genome-wide screening and functional analysis identify a large number of long noncoding RNAs involved in the sexual reproduction of rice. Genome Biol. 2014;15:512

11. Kang C, Liu Z. Global identification and analysis of long non-coding RNAs in diploid strawberry Fragaria vesca during flower and fruit development. BMC Genomics. 2015;16:815.

12. Zhu B, Yang $Y$, Ran $L$, Fu D, Wen $L$, Luo $Y$, Zhu $H$. RNA sequencing and functional analysis implicate the regulatory role of long non-coding RNAs in tomato fruit ripening. J Exp Bot. 2015;66:4483-95.

13. An N, Fan S, Wang YB, Zhang LZ, Gao C, Zhang D, Han MY. Genome-wide identification, characterization and expression analysis of long non-coding RNAs in different tissues of apple. Gene. 2018;666:44-57.

14. Zhang YC, Chen YQ. Long noncoding RNAs: new regulators in plant development. Biochem Biophys Res Commun. 2013;436:111-4.
15. Heo JB, Sung S. Vernalization-mediated epigenetic silencing by a long intronic noncoding RNA. Science. 2011:331:76-9.

16. Ding JH, Lu Q, Ouyang YD, Mao HL, Zhang PB, Yao JL, et al. A long noncoding RNA regulates photoperiod-sensitive male sterility, an essential component of hybrid rice. PNAS. 2012;109:2654-9.

17. Zhu Q, Stephen S, Taylor J, Helliwell CA, Wang MB. Long RNAs responsive to Fusarium oxysporum infection in Arabidopsis thaliana. New Phytol. 2014; 201:574-84

18. Wang JY, Yu WG, Yang YW, Li X, Chen TZ, Liu TL, et al. Genome-wide analysis of tomato long non-coding RNAs and identification as endogenous target mimic for microRNA in response to TYLCV infection. Sci Rep. 2015;5: 16946.

19. Qin T, Zhao HY, Cui P, Albesher N, Xiong LM. A nucleus-localized long noncoding RNA enhances drought and salt stress tolerance. Plant Physiol. 2017; 175:1321-36.

20. Nejat N, Ramalingam A, Mantri N. Advances in transcriptomics of plants Adv Biochem Eng Biotechnol. 2018;164:161-85.

21. Zhang CQ, Ren Y, Guo SG, Zhang HY, Gong GY, Du YC, et al. Application of comparative genomics in developing markers tightly linked to the Pm-2F gene for powdery mildew resistance in melon (Cucumis melo L.). Euphytica. 2013;190:157-68.

22. Zhu QL, Gao P, Wan Y, Cui HN, Fan C, Liu S, Luan FS. Comparative transcriptome profiling of genes and pathways related to resistance against powdery mildew in two contrasting melon genotypes. Sci Hortic. 2018;161: 169-80.

23. Yoon JH, Abdelmohsen $\mathrm{K}$, Gorospe M. Functional interactions among microRNAs and long noncoding RNAs. Semin Cell Dev Biol. 2014;34:9-14.

24. Fan $\mathrm{CY}$, Hao ZQ, Yan JH, Li GL. Genome-wide identification and functional analysis of lincRNAs acting as miRNA targets or decoys in maize. BMC Genomics. 2015:16:793.

25. Wang AH, Hu JH, Gao CB, Chen GL, Wang BC, Lin CF, et al. Genomewide analysis of long noncoding RNAs unveils the regulatory roles in the heat tolerance of Chinese cabbage (Brassica rapa ssp. chinensis). Sci Rep. 2019:9:5002.

26. Zhang H, Hu WG, Hao JL, Lv SK, Wang CY, Tong W, et al. Genome-wide identification and functional prediction of novel and fungi-responsive lincRNAs in Triticum aestivum. BMC Genomics. 2006;17:238.

27. Zhang L, Wan MJ, Li NN, Wang HL, Qiu P, Pei LL, et al. Long noncoding RNAs involve in resistance to Verticillium dahliae, a fungal disease in cotton. Plant Biotechnol J. 2018;16:1172-85.

28. Bai $Y$, Dai $X$, Harrison AP, Chen M. RNA regulatory networks in animals and plants: a long noncoding RNA perspective. Brief Funct Genomics. 2015;14:91-101.

29. Cui J, Luan YS, Jiang N, Bao H, Meng J. Comparative transcriptome analysis between resistant and susceptible tomato allows the identification of IncRNA16397 conferring resistance to Phytophthora infestans by coexpressing glutaredoxin. Plant J. 2017;89:577-89.

30. Jiang JJ, Ma SH, Ye NH, Jiang M, Cao JS, Zhang JH. WRKY transcription factors in plant responses to stresses. J Integr Plant Biol. 2017:59:86-101.

31. Appiano M, Catalano D, Santillán Martínez M, Lotti C, Zheng Z, Visser RG, et al. Monocot and dicot MLO powdery mildew susceptibility factors are functionally conserved in spite of the evolution of class-specific molecular features. BMC Plant Biol. 2015;15:257.

32. Zheng Z, Appiano M, Pavan S, Bracuto V, Ricciardi L, Visser RG, et al Genome-wide study of the tomato SIMLO gene family and its functional characterization in response to the powdery mildew Fungus Oidium neolycopersici. Front Plant Sci. 2016;7:380.

33. Kuhn H, Lorek J, Kwaaitaal M, Consonni C, Becker K, Micali C, et al. Key components of fifferent plant defense pathways are dispensable for Pwdery mildew resistance of the Arabidopsis m/o2 mlo6 mlo12 triple mutant. Front Plant Sci. 2017:8:1006.

34. Cesana M, Cacchiarelli D, Legnini I, Santini T, Sthandier O, Chinappi M, et al. A long noncoding RNA controls muscle differentiation by functioning as a competing endogenous RNA. Cell. 2011;147:358-36.

35. Li Y, Cao XL, Zhu Y, Yang XM, Zhang KN, Xiao ZY, et al. Osa-miR398b boosts $\mathrm{H}_{2} \mathrm{O}_{2}$ production and rice blast disease-resistance via multiple superoxide dismutases. New Phytol. 2019;222:1507-22.

36. Camargo-Ramírez R, Val-Torregrosa B, San SB. MiR858-mediated regulation of flavonoid-specific MYB transcription factor genes controls resistance to pathogen infection in Arabidopsis. Plant Cell Physiol. 2018;59:190-204.

37. Cohen R. A leaf disk assay for detection of resistance of melons to Sphaerotheca fuliginea race 1. Plant Dis. 1993;77:135-44. 
38. Trapnell C, Roberts A, Goff L, Pertea G, Kim D, Kelley DR, et al. Differential gene and transcript expression analysis of RNA-Seq experiments with TopHat and cufflinks. Nat Protoc. 2012;7:562-78.

39. Tafer H, Hofacker IL. RNAplex: a fast tool for RNA-RNA interaction search. Bioinformatics. 2008;24:2657-63.

40. Young MD, Wakefield MJ, Smyth GK, Oshlack A. Gene ontology analysis for RNA-seq: accounting for selection bias. Genome Biol. 2010;11:R14.

41. Dai X, Zhao PX. psRNATarget: a plant small RNA target analysis server. Nucleic Acids Res. 2011;39:155-9.

42. Wu HJ, Wang ZM, Wang M, Wang XJ. Wide-spread long noncoding RNAs as endogenous target mimics for microRNAs in plants. Plant Physiol. 2013;161: 1875-84.

\section{Publisher's Note}

Springer Nature remains neutral with regard to jurisdictional claims in published maps and institutional affiliations.

Ready to submit your research? Choose BMC and benefit from:

- fast, convenient online submission

- thorough peer review by experienced researchers in your field

- rapid publication on acceptance

- support for research data, including large and complex data types

- gold Open Access which fosters wider collaboration and increased citations

- maximum visibility for your research: over $100 \mathrm{M}$ website views per year

At BMC, research is always in progress.

Learn more biomedcentral.com/submissions 